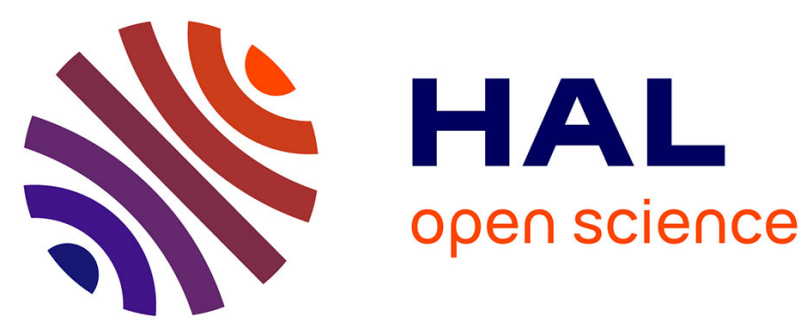

\title{
Study of Rb atomic transitions D 1,2 lines in strong magnetic field based on fluorescence spectra of sub-micron thin cell.
}

G. Hakhumyan, Claude Leroy, Y. Pashayan-Leroy, D. Sarkisyan

\section{- To cite this version:}

G. Hakhumyan, Claude Leroy, Y. Pashayan-Leroy, D. Sarkisyan. Study of Rb atomic transitions D 1,2 lines in strong magnetic field based on fluorescence spectra of sub-micron thin cell.. International Conference on Laser Physics 2010, Oct 2010, Ashtarak, Armenia. pp.79980V, 10.1117/12.890893 . hal-00620533

\section{HAL Id: hal-00620533 https://hal.science/hal-00620533}

Submitted on 7 Sep 2011

HAL is a multi-disciplinary open access archive for the deposit and dissemination of scientific research documents, whether they are published or not. The documents may come from teaching and research institutions in France or abroad, or from public or private research centers.
L'archive ouverte pluridisciplinaire HAL, est destinée au dépôt et à la diffusion de documents scientifiques de niveau recherche, publiés ou non, émanant des établissements d'enseignement et de recherche français ou étrangers, des laboratoires publics ou privés. 


\title{
Study of $\mathbf{R b}$ atomic transitions $\mathrm{D}_{1,2}$ lines in strong magnetic field based on fluorescence spectra of sub - micron thin cell
}

\author{
G. Hakhumyan ${ }^{1,2}$, A.Sargsyan ${ }^{1}$, C. Leroy ${ }^{2}$, Y. Pashayan-Leroy ${ }^{2}$, D. Sarkisyan ${ }^{1}$ \\ ${ }^{1}$ Institute for Physical Research, NAS of Armenia, Ashtarak, 0203, Armenia \\ ${ }^{2}$ Laboratoire Interdisciplinaire Carnot de Bourgogne, UMR CNRS 5209 - Université de Bourgogne, \\ F-21078 Dijon Cedex, France
}

\begin{abstract}
The so-called " $\lambda / 2$-Zeeman technique" (HLZT) for studies of individual optical transition between Zeeman sublevels of atomic hyperfine structure in an external magnetic field of $B=10-2500 \mathrm{G}$ is presented. Particularly, implementation of HLZT allows one to realize a direct determination of frequency shift and a strong modification of an optical transition probability between Zeeman components in a $B$-field. The main advantages of the method compared to " $\lambda$-Zeeman technique" (LZT) is that it allows one to study weak transitions. Particularly, with the help of fluorescence on ${ }^{87} \mathrm{Rb}, \mathrm{D}_{2}$ line, $F_{g}=1 \rightarrow F_{e}=3$ transitions, three "forbidden" transitions in magnetic field $B$ are detected and studied. Also, on ${ }^{87} \mathrm{Rb}$ $\mathrm{D}_{1}$ line, $F_{g}=1, m_{F}=0 \rightarrow F_{e}=1, m_{F}=0$ "forbidden" transition is detected when $B \sim 400 \mathrm{G}$. A strong modification of the probability for these "forbidden" transitions is revealed. The theoretical model well describes the observed results.
\end{abstract}

\section{INTRODUCTION}

The development of atomic vapor cells of sub-micrometric thickness (STC) opens a new possibility to realize subDoppler spectroscopy. Interaction of atoms with laser radiation field in such cells is strictly anisotropic. Atoms with nonzero longitudinal velocity projection experience frequent quenching collisions with the cell walls (windows). As a result, the interaction is favored for the atoms with zero longitudinal velocity, i.e. those yielding sub-Doppler fluorescence response ${ }^{1-4}$. With the proper choice of parameters, it is possible to achieve a sevenfold narrowing of the spectrum.

It is well-known that atomic transitions of alkali metals split in magnetic fields into Zeeman components, whose frequency shifts deviate from the linear behavior in already moderate magnetic fields. As this takes place, the probabilities of atomic transitions usually change significantly as well ${ }^{5-7}$. Previous papers ${ }^{8-11}$ demonstrated that the use of resonance fluorescence spectra of a STC filled with atomic vapor and of thickness $L=0.5 \lambda$ (where $\lambda=794 \mathrm{~nm}$ (or 780 $\mathrm{nm}$ ) is the wavelength of the laser radiation whose frequency is resonant with the atomic transition of the $\mathrm{D}_{1,2}$ lines of $\mathrm{Rb}$ ) allows one to separate and study atomic transitions between the levels of the hyperfine structure of the $\mathrm{D}_{1,2}$ lines of ${ }^{87} \mathrm{Rb}$ atoms in magnetic fields with $B$ varying in the range of $10-200 \mathrm{G}$. The achieved high sub-Doppler spectral resolution is caused by the effect of a strong narrowing of the fluorescence spectrum of a nanocell with the atomic vapor column thickness $L=0.5 \lambda$ (the method was called HLZM) compared to normal $1 \mathrm{~cm}$-long cells (for which the Doppler width is $\sim 500 \mathrm{MHz}$ ). It is known that, with the saturated absorption (SA) technique, the sub-Doppler spectral resolution can also be achieved using cm-long cells (when the parameters are properly chosen, it is possible to obtain peaks of reduced absorption with a width close to the natural one $\sim 6 \mathrm{MHz}$ ). In the publications ${ }^{12,13}$, the SA technique is used to study spectra of $D_{1}$ and $D_{2}$ lines of $R b$ atoms. One of the significant disadvantages of the application of the SA technique is the presence of so-called cross-over resonances in the spectra. In a magnetic field, these resonances split into numerous components, making the spectrum very difficult to process. This restricts the magnitude of acceptable magnetic fields and, as a rule, $B$ should be below $100 \mathrm{G}$. Another significant disadvantage is the fact that SA is strongly nonlinear and, therefore, the peak amplitudes of the decreased absorption do not correspond to the probability of atomic transitions at which frequencies these peaks are formed. This additionally complicates the processing of spectra. At the same time, using the HLZM technique, the peak value of fluorescence (up to laser intensities of $\sim 20 \mathrm{~mW} / \mathrm{cm}^{2}$ ) corresponds to the probability of atomic transitions. This allows one to directly study the dependence of the probability on $B$. Another important advantage of the HLZM method is the possibility of using strong permanent magnets (PMs) that can generate fields of several thousand gausses at distances of several centimeters. The fields of these PMs are 
strongly non-homogeneous and the gradient can achieve $\sim 100-200 \mathrm{G} / \mathrm{mm}$, which makes impossible to use cm-long cells. At the same time, due to the small thickness of the STC $(\sim 400 \mathrm{~nm})$, the gradient of $B$ inside the STC is four to five orders of magnitude smaller than the measured value of $B$. This remarkable feature of a STC is used for the study of fluorescence spectra ${ }^{14}$ as well as for absorption spectra of atomic vapors within $B$ fields from $10-2500 \mathrm{G}$ at the vapor column thickness $L=\lambda$ (the method was called LZM) ${ }^{15}$.

The aim of this work is to experimentally demonstrate that with the help of HLZM it is possible to study the "forbidden" transitions on $\mathrm{D}_{1,2}$ lines of the ${ }^{87} \mathrm{Rb}$. Also, a theoretical consideration is provided.

\section{EXPERIMENTAL PART}

Sketch of the experimental setup is presented in Fig.1a. The circularly polarized light of extended cavity diode laser (ECDL, $\lambda=780 \mathrm{~nm}, P_{L} \sim 30 \mathrm{~mW}, \gamma_{L}<1 \mathrm{MHz}$ ) resonant with ${ }^{87} \mathrm{Rb} \mathrm{D}_{2}$ transition frequency, after passing through Faraday isolator is directed onto the Rb STC 2 with the thickness of vapor column $L=\lambda / 2$, at an angle close to normal. The needed temperature regime of the STC $\left(\mathrm{T}_{\mathrm{SA}} \sim 110-120^{\circ} \mathrm{C}, \mathrm{T}_{\mathrm{W}} \sim 140-150{ }^{\circ} \mathrm{C}\right.$, corresponding to $N \sim 6 \times 10^{12}-1.5 \times$ $10^{13}$ atoms $/ \mathrm{cm}^{3}$ ) is provided by a special oven with 3 openings: 2 inlets for laser beam passage and one orthogonal inlet for side fluorescence detection. This geometry allows simultaneous recording of fluorescence and transmission spectra.
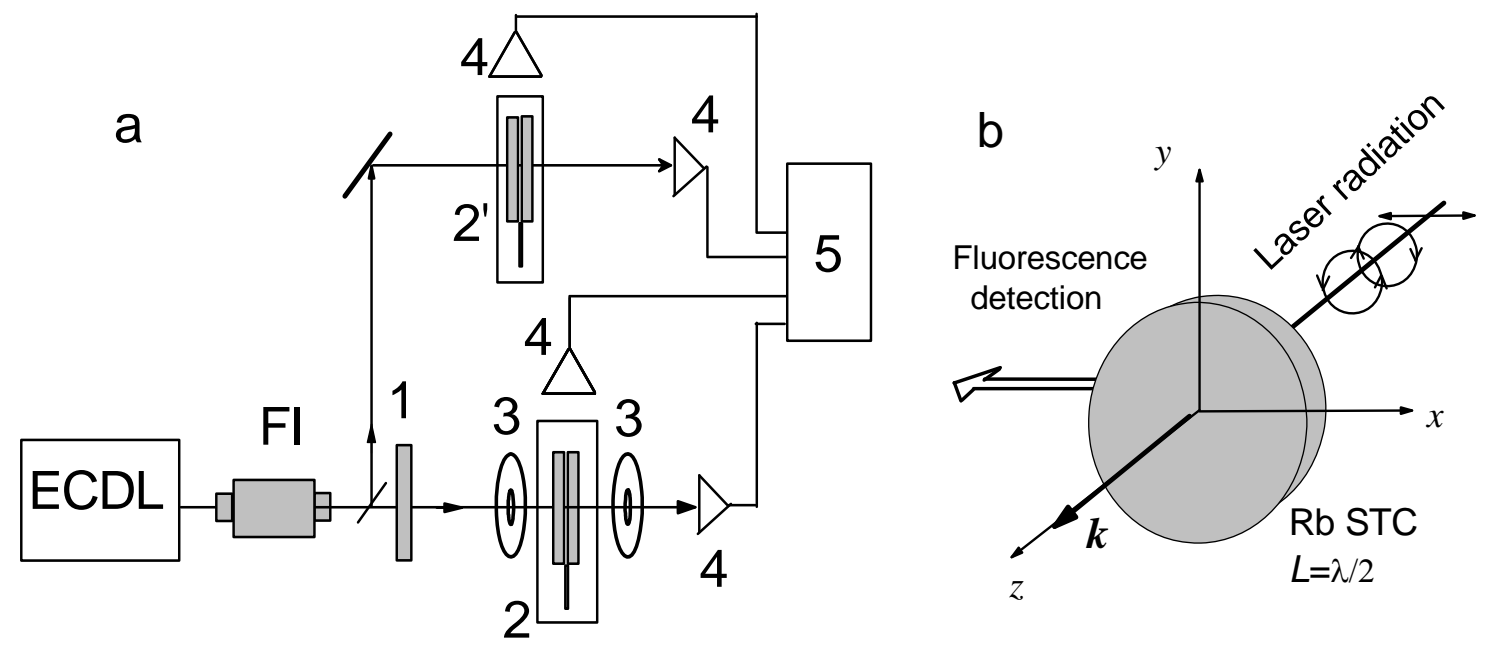

Fig. 1 a - Sketch of the experimental setup. FI - Faraday isolator, 1 - $\lambda / 4$ plate, 2 - STC and the oven, 2'- the auxiliary STC and the oven, 3 - RMs, 4 - photodetectors, 5 - digital storage oscilloscope; $b$ - mutual orientations of laser excitation and detection of fluorescence emitted by a STC of $L=\lambda / 2$.

The fluorescence signal is detected by a photodiode 4 with an aperture of $1 \mathrm{~cm}^{2}$ which is placed at $90^{\circ}$ angle of the laser propagation direction. The photodiode signal is recorded by a digital four channel storage oscilloscope, Tektronix TDS 2014B 5. A Glan prism is used to purify initial linear radiation polarization of the laser radiation; to produce a circular polarization a $\lambda / 4$ plate 1 is utilized. The geometrical configuration of the experiment is shown in Fig.1b. Magnetic field is directed along the laser radiation propagation direction $\boldsymbol{k}(\boldsymbol{B} / / \boldsymbol{k})$.

With the help of a beam splitter, 50\% of the pump beam is directed on the auxiliary 2' STC with Rb used as a frequency reference for $B=0$. In some cases the reference spectrum is transmission spectrum ${ }^{15}$ obtained with an auxiliary STC of thickness $L=\lambda$. The small longitudinal magnetic field $(B<250 \mathrm{G})$ is applied to the STC by a system of Helmholtz coils (not shown in Fig.1a). The $B$-field strength is measured by a calibrated Hall gauge. Among the advantages of HLZM is the possibility to apply much stronger magnetic field using widely available strong PMs. In spite of strong inhomogeneity of $B$-field (in our case it can reach $150 \mathrm{G} / \mathrm{mm}$ ), due to small thickness of the atomic vapor column inside 
STC $(L=390 \mathrm{~nm})$ the variation of $B$ inside the column is less than $0.1 \mathrm{G}$, i.e. by several orders less than the applied $B$ value.

\section{Implementation of HLZT method for the ${ }^{87} \mathrm{Rb} \mathrm{D}_{2}$ line $F_{g}=1 \rightarrow F_{e}=3$ forbidden transitions study}

The optical transitions between magnetic sublevels of hf states for ${ }^{87} \mathrm{Rb} \mathrm{D}_{2}$ line in the case of $\sigma^{+}$(left circular) polarized excitation and selection rules $\Delta m_{F}=+1$ are depicted in Fig.2.

Examination of the fluorescence peak formed in a STC allow one to obtain, identify and investigate the atomic transitions between the Zeeman sublevels in the transmission spectrum of the ${ }^{87} \mathrm{Rb} \mathrm{D}_{2}$ line in the range of magnetic fields $10-2500 \mathrm{G}$, i.e., the transitions $F_{g}=1 \rightarrow F_{e}=0, F_{g}=1 \rightarrow F_{e}=1, F_{g}=1 \rightarrow F_{e}=2$ as well as $F_{g}=1 \rightarrow F_{e}=3$ for $\sigma^{+}$ polarizations of the exciting laser radiation. It is revealed experimentally that in relatively weak magnetic fields (about $100 \mathrm{G})$ with the $\sigma^{+}$-polarized laser also those atomic transitions are recorded, for which new selection rules with respect to the quantum number $F$ take place: $\Delta F=2, \Delta m_{F}=+1$. The probability of these transitions increases with the increase of the magnetic field strength. Note that for $B=0$ the $\Delta F=2$ transitions are strongly forbidden. In the field of $B \sim 200 \mathrm{G}$ the probability of the transition $F_{g}=1, m_{F}=-1 \rightarrow F_{e}=3, m_{F}=0$ (Fluorescence peak 7) becomes equal to the probability of the transition $F_{g}=1, m_{F}=+1 \rightarrow F_{e}=2, m_{F}=+2$ (Fluorescence peak 6). Also with a field $B>400 \mathrm{G}$ the transitions $F_{g}=$ $1, m_{F}=0 \rightarrow F_{e}=3, m_{F}=+1$ (Fluorescence peak 8) and $F_{g}=1, m_{F}=+1 \rightarrow F_{e}=3, m_{F}=+2$ (Fluorescence peak 9) are easily recorded.

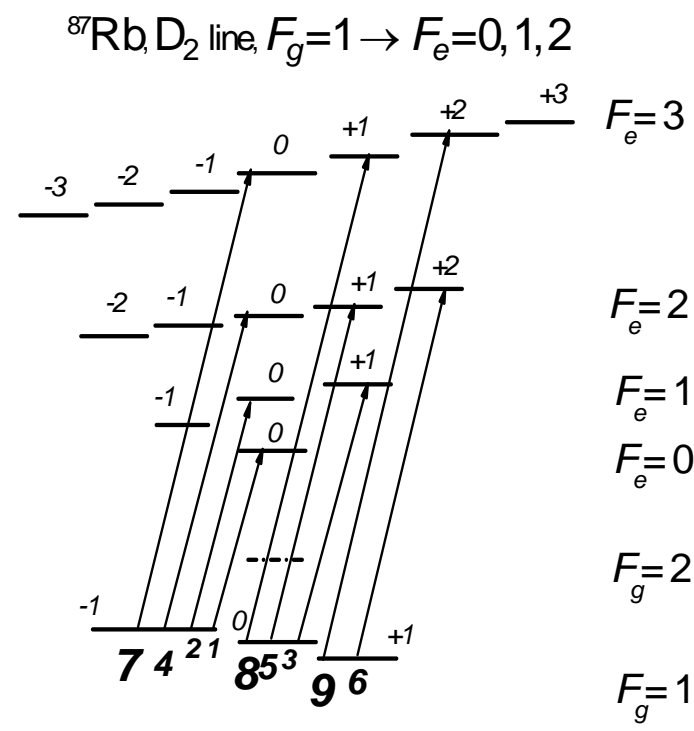

Fig. 2. Energy level diagram of $\mathrm{D}_{2}$ line of ${ }^{87} \mathrm{Rb}$ in a magnetic field and possible atomic transitions for $\sigma^{+}-$ polarized excitation.

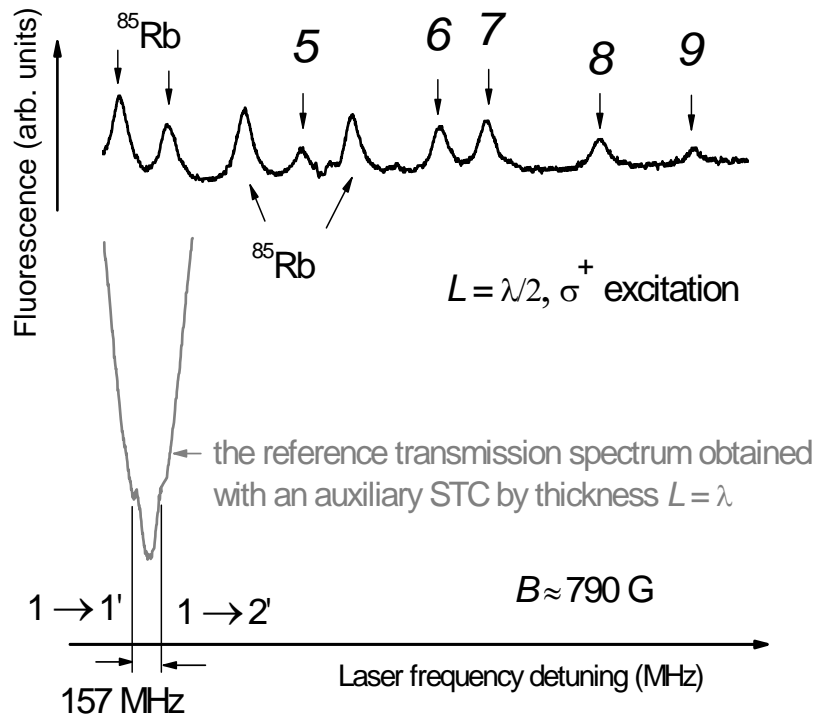

Fig. 3. Fluorescence spectrum of $\mathrm{D}_{2}$ line of the ${ }^{87} \mathrm{Rb}, F_{g}=1 \rightarrow F_{e}=$ 2, 3 transitions. Fluorescence peaks under number 7, 8, 9 represent atomic transitions which have zero probability for $B=0$. The lower curve-reference transmission spectrum is obtained with an auxiliary STC of thickness $L=\lambda$.

Figure 3 shows the fluorescence spectrum of STC for the atomic $F_{g}=1 \rightarrow F_{e}=2,3$ transitions. The fluorescence peaks under number 7, 8, 9 represent forbidden (when $B=0$ ) atomic transitions $F_{g}=1 \rightarrow F_{e}=3$. Note, that the amplitudes of the Fluorescence peaks 7, 8, 9 are comparable to the peak numbered $6\left(F_{g}=1, m_{F}=+1 \rightarrow F_{e}=2, m_{F}=+2\right)$ which is the strongest atomic transition when $B=0$. Moreover, the amplitude of the fluorescence peak 9 is even larger than that of the 
fluorescence peak numbered 6. It is important to note, that both the frequency position as well as the fluorescence peaks 6, 7, 8 and 9 are well described by the theoretical curves presented in Figures 5 and 6 in a previous work ${ }^{15}$. Thus, HLZT is a convenient and robust tool to study forbidden atomic transitions in an external magnetic field.

\section{Use of HLZT method for the ${ }^{87} \mathrm{Rb} \mathrm{D}_{1}$ line $F_{g}=1, m_{F}=0 \rightarrow F_{e}=1 m_{F}=0$ forbidden transition study}

In order to study the ${ }^{87} \mathrm{Rb} \mathrm{D}_{1}$ line $F_{g}=1, m_{F}=0 \rightarrow F_{e}=1 m_{F}=0$ forbidden transition we use a $\pi$-polarized excitation (selection rules $\Delta m_{F}=0$ are depicted in Fig.4). For this case the magnetic field $B$ is directed along the laser electric field

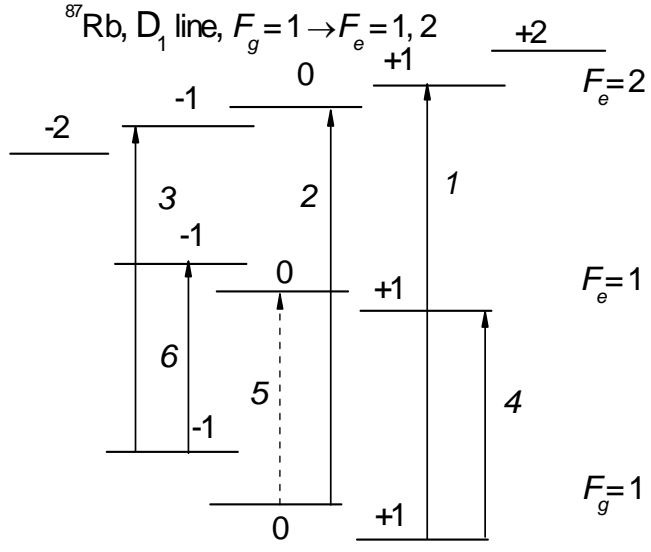

Fig.4. Energy level diagram of $\mathrm{D}_{1}$ line of ${ }^{87} \mathrm{Rb}$ in a magnetic field and possible atomic transitions for $\pi$-polarized excitation. Transition number 5 is forbidden when $B=0$.

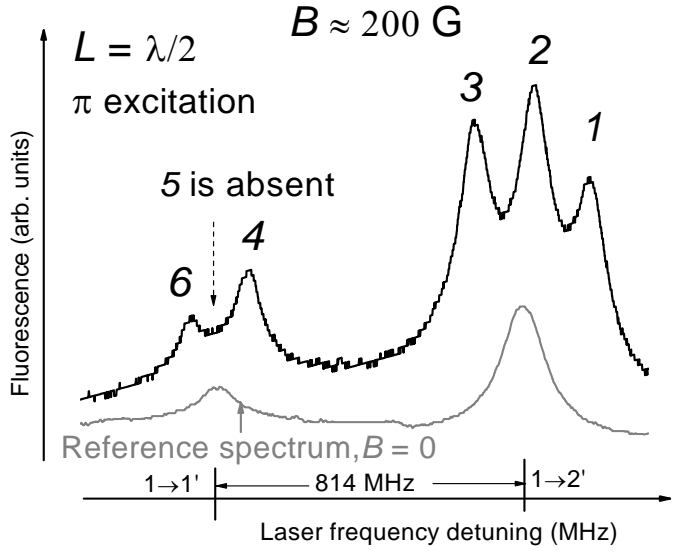

Fig.5. Fluorescence spectrum of $\mathrm{D}_{1}$ line of the ${ }^{87} \mathrm{Rb}, F_{g}$ $=1 \rightarrow F_{e}=1,2$ transitions, $B \approx 200 \mathrm{G}$. Fluorescence peaks under number $1,2,3,4,5$ and 6 represent atomic transitions shown in diagram Fig.4. The lower curvereference fluorescence spectrum is obtained with the auxiliary STC of thickness $L=\lambda / 2$ for $B=0$.

$\boldsymbol{E}(\boldsymbol{B} / / \boldsymbol{E})$ and the fluorescence detection is in the direction perpendicular to $\boldsymbol{E}$ (see Fig.1b).

Fig.5 (the upper curve) shows the fluorescence spectrum of STC for the atomic ${ }^{87} \mathrm{Rb}, F_{g}=1 \rightarrow F_{e}=1,2$ transitions when $B \approx 200 \mathrm{G}$. The lower grey curve-reference fluorescence spectrum obtained with the auxiliary STC of thickness $L=\lambda / 2$. As it is seen all the five allowed transitions are well seen, while forbidden transition 5 is absent. For $B=0$ the ratio of 1 , 2, 3 amplitudes is 3:4:3 and for 4, 6 amplitudes is 1:1 (see Fig.6). As it is seen for the magnetic field $B \approx 200 \mathrm{G}$ there already occur modifications of the amplitudes.

Fig.6 (the upper curve) presents the fluorescence spectrum of STC for the atomic ${ }^{87} \mathrm{Rb}, F_{g}=1 \rightarrow F_{e}=1,2$ transitions when $B \approx 410 \mathrm{G}$. The lower grey curve is the reference fluorescence spectrum obtained with the auxiliary STC of thickness $L=\lambda / 2$ for $B=0$. As it is seen all the six transitions are well seen, including forbidden (when $B=0$ ) $\operatorname{transition}$ $F_{g}=1, m_{F}=0 \rightarrow F_{e}=1 m_{F}=0$ numbered 5. Thus, a strong modification of atomic transitions in magnetic field is seen. It is important to note, that both the frequency position as well as the fluorescence peaks 1, 2, 3, 4, 5 and 6 are well described by the theoretical curves presented in Figures 7 and 8. Particularly, the experimentally detected frequency shift, from the initial position when $B=0$, for the components $1,2,3,4$ and 6 is well described by the theoretical curves shown in Fig.7. Note, that the component 5 has practically zero shift from the initial frequency position $F_{g}=1 \rightarrow F_{e}=1$. As to the modification of the probability of the component 5 as a function of $B$ value, as it is predicted theoretically by the curves presented in Fig.8, for $B>400 \mathrm{G}$ it's probability becomes larger than that of the component 6 . This is well seen from the experimental curves shown in Fig.6. 


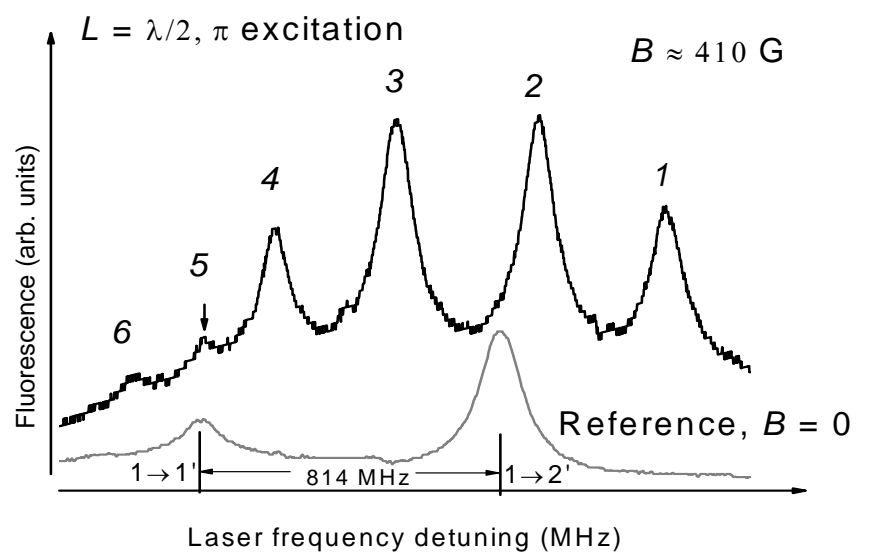

Fig.6. Fluorescence spectrum of $\mathrm{D}_{1}$ line of the ${ }^{87} \mathrm{Rb}, F_{g}=1 \rightarrow F_{e}=1,2$ transitions, $B \approx 410$ G. Fluorescence peaks $1,2,3,4,5$ and 6 represent atomic transitions shown in diagram Fig.4. As it is seen the fluorescence peak under number 5 already exists. The lower grey curve-reference fluorescence spectrum is obtained with an auxiliary STC of thickness $L=\lambda / 2$ for $B=0$.

\section{Theoretical model}

When an external magnetic field is applied, the Hamiltonian for the atom in the presence of a magnetic field can be expressed as the sum of the unperturbated atomic Hamiltonian $\boldsymbol{H}_{0}$ and the so-called Zeeman Hamiltonian $\boldsymbol{H}_{Z}$ :

$$
\boldsymbol{H}=\boldsymbol{H}_{0}+\boldsymbol{H}_{Z}
$$

The Hamiltonian of interaction $\boldsymbol{H}_{Z}$ may be expanded using different forms ${ }^{5,70}$, in this way ${ }^{5} \boldsymbol{H}_{Z}$ may be expressed as

$$
\boldsymbol{H}_{Z}=\left(-\frac{\mu_{B}}{\hbar}\right) \boldsymbol{B} \cdot\left(L+g_{S} \boldsymbol{S}+g_{I} /\right)
$$

where $\boldsymbol{L}$ is the orbital angular momentum, $\boldsymbol{S}$ is the spin momentum and $\boldsymbol{I}$ the nuclear spin momentum, $\mu_{B}$ is the Bohr magneton and $g_{S}, g_{I}$ are respectively the spin and nuclear Landé factor.

We adopt a matrix representation in the coupled basis, that is, the basis of the unperturbated atomic state vectors $\left|(n=3), L, J, F, m_{F}\right\rangle$ to evaluate the matrix elements of the Hamiltonian (1). In this basis, the diagonal matrix elements are given by

$$
\left\langle F, m_{F}|\boldsymbol{H}| F, m_{F}\right\rangle=E_{0}(F)+\mu_{B} g_{F} m_{F} B_{Z}
$$

where $E_{0}(F)$ is the initial energy of the sublevel $\left|(n=3), L, J, F, m_{F}\right\rangle \equiv\left|F, m_{F}\right\rangle$ and $g_{F}$ is the effective Landé factor.

The off-diagonal matrix elements are non-zero for levels verifying the selection rules $\Delta L=0, \Delta J=0, \Delta F= \pm 1, \Delta m_{F}=0$ 


$$
\begin{aligned}
\left\langle F-1, m_{F}|\boldsymbol{H}| F, m_{F}\right\rangle & =\left\langle F, m_{F}|\boldsymbol{H}| F-1, m_{F}\right\rangle=-\frac{\mu_{B} B_{Z}}{2}\left(g_{J}-g_{I}\right) \\
& \times\left(\frac{\left[(J+I+1)^{2}-F^{2}\right]\left[F^{2}-(J-I)^{2}\right]}{F}\right)^{1 / 2}\left(\frac{F^{2}-m_{F}^{2}}{F(2 F+1)(2 F-1)}\right)^{1 / 2} .
\end{aligned}
$$

The Hamiltonian matrix is then block diagonal where each block corresponds to a given value of $m_{F}$.

The diagonalization of the Hamiltonian matrix allows one to find the eigenvectors and the eigenvalues, that is to determine the eigenvalues corresponding to the energies of Zeeman sublevels and the new states vectors which can be expressed in terms of the initial unperturbed atomic state vectors,

$$
\left|\Psi\left(F_{e}^{\prime}, m_{F_{e}}\right)\right\rangle=\sum_{F_{e}=I-J_{e}}^{F_{e}=I+J_{e}} \alpha_{F_{e}^{\prime} F_{e}}^{e}\left(B_{z}, m_{F_{e}}\right)\left|F_{e}, m_{F_{e}}\right\rangle,
$$

and

$$
\left|\Psi\left(F_{g}^{\prime}, m_{F_{g}}\right)\right\rangle=\sum_{F_{g}=I-J_{g}}^{F_{g}=I+J_{g}} \alpha_{F_{g}^{\prime} F_{g}}^{g}\left(B_{z}, m_{F_{g}}\right)\left|F_{g}, m_{F_{g}}\right\rangle .
$$

The state vectors $\left|F_{e}, m_{e}\right\rangle$ and $\left|F_{g}, m_{g}\right\rangle$ are the unperturbated state vectors, respectively, for the excited and the ground states. The coefficients $\alpha_{F_{e}^{\prime} F_{e}}^{e}\left(B_{z}, m_{F_{e}}\right)$ and $\alpha_{F_{g}^{\prime} F_{g}}^{g}\left(B_{z}, m_{F_{g}}\right)$ are mixing coefficients, respectively, for the excited and the ground states; they depend on the field strength and magnetic quantum numbers $m_{e}$ or $m_{g}$.

The probability of a transition, induced by the interaction of the atomic electric dipole and the oscillating laser electric field is proportional to the spontaneous emission rate of the associated transition $A_{e g}$, that is, to the square of the transfer coefficients modified by the presence of the magnetic field

$$
\frac{8 \pi^{2}}{3 \varepsilon_{0} \hbar \lambda_{e g}^{3}}\left|\left\langle e\left|D_{q}\right| g\right\rangle\right|^{2}=A_{e g} \propto a^{2}\left[\Psi\left(F_{e}^{\prime}, m_{F_{e}}\right) ; \Psi\left(F_{g}^{\prime}, m_{F_{g}}\right) ; q\right],
$$

where $D_{q}$ denote the standard components of the electric dipole moment:

$$
\boldsymbol{D} \cdot \boldsymbol{e}=\sum_{q} D_{q} e_{q}
$$

with $q=-1,0,1$.

In eq. (7), the transfer coefficients are expressed as

$$
\left.a \mid \Psi\left(F_{e}^{\prime}, m_{F_{e}}\right) ; \Psi\left(F_{g}^{\prime}, m_{F_{g}}\right) ; q\right]=\sum_{F_{e}, F_{g}} \alpha_{F_{e} F_{e}}^{e}\left(B_{z}, m_{F_{e}}\right) a\left(F_{e}, m_{F_{e}} ; F_{g}, m_{F_{g}} ; q\right) \alpha_{F_{g} F_{g}}^{g}\left(B_{z}, m_{F_{g}}\right),
$$

where the unperturbated transfer coefficients have the following definition 


$$
\begin{aligned}
a\left(F_{e}, m_{F_{e}} ; F_{g}, m_{F_{g}} ; q\right)= & (-1)^{1+++J_{e}+F_{e}+F_{g}-m_{F_{e}}} \\
& \times \sqrt{2 J_{e}+1} \sqrt{2 F_{e}+1} \sqrt{2 F_{g}+1}\left(\begin{array}{ccc}
F_{e} & 1 & F_{g} \\
-m_{F_{e}} & q & m_{F_{g}}
\end{array}\right)\left\{\begin{array}{ccc}
F_{e} & 1 & F_{g} \\
J_{g} & I & J_{e}
\end{array}\right\},
\end{aligned}
$$

the parenthesis and curly brackets denote, respectively, the $3-j$ and $6-j$ symbols, $g$ and $e$ point respectively ground and excited states.

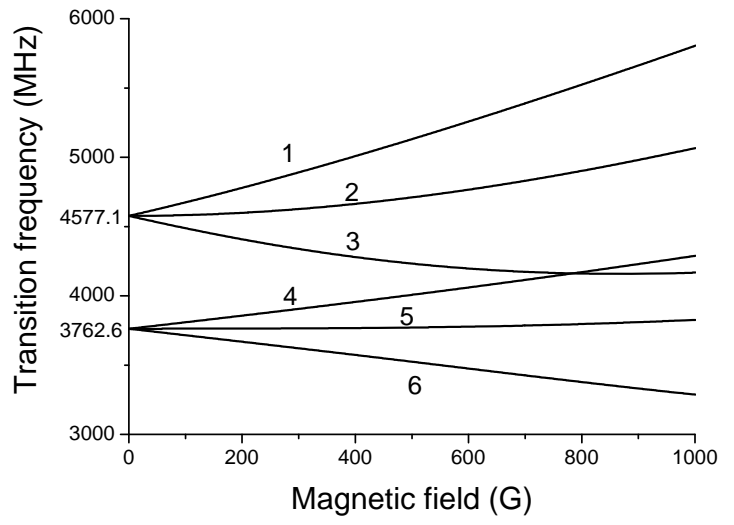

Fig.7. Theoretical values of the frequency position of atomic transitions in ${ }^{87} \mathrm{Rb} \mathrm{D}_{1}$ line $1,2,3,4,5$ and 6 components for $\pi$-polarization of laser radiation versus the magnetic field (numbers denote the corresponding transitions, see Fig.4).

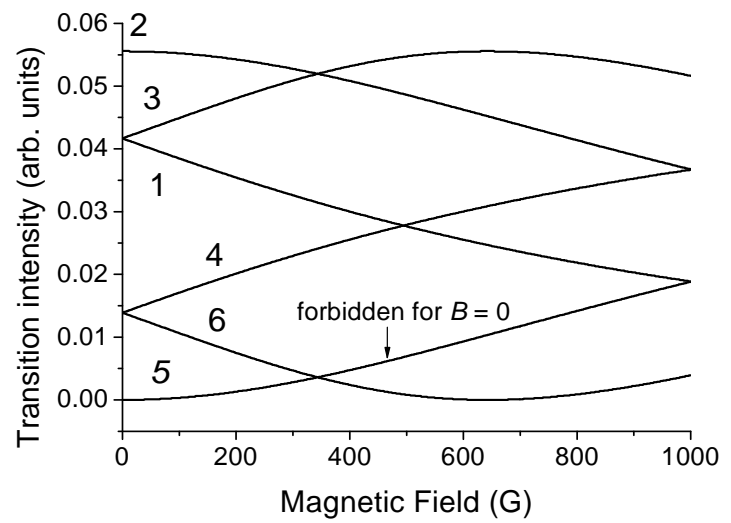

Fig.8. Theoretical values of the probability amplitudes (or line intensities) for atomic transitions of the ${ }^{87} \mathrm{Rb}, F_{g}=1$ $\rightarrow F_{e}=1,2$, with $\pi$-polarization of laser radiation versus the magnetic field.

\section{Conclusion}

The " $\lambda / 2$-Zeeman technique" (HLZT) is shown to be a convenient and robust tool for the study of individual transitions between the Zeeman sublevels of hyperfine levels in an external magnetic field of $10-2500$ G. HLZT is based on resonant fluorescence spectrum emitted by a STC with $L=\lambda / 2$, where $\lambda$ is the resonant wavelength $\left(780 \mathrm{~nm}\right.$ for $\mathrm{D}_{2}$ and $794 \mathrm{~nm}$ for $\mathrm{D}_{1}$ line of $\mathrm{Rb}$ ). Narrow fluorescence peak of spectral width $\sim 70 \mathrm{MHz}$ is split into several components in a magnetic field; their frequency positions and probabilities depend on the B-field. The implementation of HLZT allows us to reveal strong modification of the probability of the atomic transition. Also, the HLZT allows us to detect strong modification of three "forbidden" transitions with the help of fluorescence on ${ }^{87} \mathrm{Rb}, \mathrm{D}_{2}$ line, $F_{g}=1 \rightarrow F_{e}=3$ transitions. On ${ }^{87} \mathrm{Rb}, \mathrm{D}_{1}$ line, $F_{g}=1, m_{F}=0 \rightarrow F_{e}=1, m_{F}=0$ "forbidden" transition probability also demonstrates a strong modification for the magnetic field $B>300 \mathrm{G}$. The experimental results are in a good agreement with the theoretical values.

\section{Acknowledgement}

The authors are grateful to A. Sarkisyan for his valuable participation in fabrication of the STC as well as to A. Papoyan for useful discussions. Research conducted in the scope of the International Associated Laboratory IRMAS. Armenian team thanks for the ANSEF PS 1868 support. 


\section{References}

[1] D. Sarkisyan, D. Bloch, A. Papoyan and M. Ducloy, "Sub-Doppler spectroscopy by sub-micron thin Cs vapour layer", Opt. Commun. 200, 201 - 208 (2001).

[2] G. Dutier, A. Yarovitski, S. Saltiel, A. Papoyan, D. Sarkisyan, D. Bloch and M. Ducloy, "Collapse and revival of a Dicke-type coherent narrowing in a sub-micron thick vapor cell transmission spectroscopy”, Europhys. Lett. 63 (1), $35-41(2003)$.

[3] D. Sarkisyan, T. Becker, A. Papoyan, P. Thoumany and H. Walther, "Sub-Doppler Fluorescence on atomic $\mathrm{D}_{2}$ line of Sub-Micron Rubidium Vapor Layer", Appl. Phys. B 76, N6, pp. 625 - 631 (2003).

[4] D. Sarkisyan, T. Varzhapetyan, A. Sarkisyan, Yu. Malakyan, A. Papoyan, A. Lezama, D. Bloch and M. Ducloy, "Spectroscopy in an extremely thin vapor cell: Comparing the cell-length dependence in fluorescence and in absorption techniques", Phys. Rev. A 69, 065802 (4p.) (2004).

[5] P. Tremblay, A. Michaud, M. Levesque, S. Theriault, M. Breton, J. Beaubien and N. Cyr, "Absorption profiles of alkali-metal D lines in the presence of a static magnetic field", Phys. Rev. A 42, 2766 - 2773 (1990).

[6] N. Papageorgiou, A. Weis, V. Sautenkov, D. Bloch and M. Ducloy, "High-resolution selective reflection spectroscopy in intermediate magnetic fields", Appl. Phys. B 59, 123 - 126 (1994).

[7] A. Papoyan, D. Sarkisyan, K. Blush, M. Auzinsh, D. Bloch and M. Ducloy, "Magnetic field-induced mixing of hyperfine states of the Cs 6P level observed with a submicron vapor cell", Laser Physics 13, 1467 - 1477 (2003).

[8] D. Sarkisyan, A. Papoyan, T. Varzhapetyan, K. Blush and M. Auzinsh, "Hyperfine structure Zeeman effect on of an atomic D1 line of a sub-micron ${ }^{87} \mathrm{Rb}$ vapor layer", Opt. and Spectrosc. 96, 328 - 334 (2004).

[9] D. Sarkisyan, A. Papoyan, T. Varzhapetyan, K. Blush and M. Auzinsh, "Fluorescence of rubidium in a submicrometer vapor cell: spectral resolution of atomic transitions between Zeeman sublevels in a moderate magnetic field", J. Opt. Soc. Am. B 22, 88 - 95 (2005).

[10] D. Sarkisyan, A. Papoyan, T. Varzhapetyan, J. Alnis, K. Blush and M. Auzinsh, "Sub-Doppler spectroscopy of Rb atoms in a sub-micron vapor cell in the presence of a magnetic field", J. Opt. A: Pure and Appl. Opt. 6, S142 S150 (2004).

[11] T.S. Varzhapetyan, G.T. Hakhumyan, V.V. Babushkin, D.H. Sarkisyan, A. Atvars and M. Auzinsh, "Study of atomic spectral lines in a magnetic field with use of a nanocell with the thickness $L=\lambda$ ", J. of Contemp. Phys. (Arm. Acad. of Sci.) 42, 223 - 229 (2007).

[12] M.U. Momeen, G. Rangarajan and P.C. Deshmukh, "Variations of intensity in Rb $\mathrm{D}_{2}$ line at weak/intermediate fields", J. Phys. B: At. Mol. Opt. Phys. 40, 3163 - 3172 (2007).

[13] G.Školnik, N.Vujičić, T. Ban, "Optical pumping of Zeeman components in rubidium vapor”, Opt. Commun..282, 1326 (2009).

[14] G. Hakhumyan, D. Sarkisyan, A. Sargsyan, A. Atvars, and M. Auzinsh, "Investigation of Rb D1 Atomic Lines in Strong Magnetic Fields by Fluorescence from a Half-Wave-Thick Cell”, Optics and Spectroscopy, 108, 685-692 (2010)

[15] A. Sargsyan, G. Hakhumyan, A. Papoyan, D. Sarkisyan, A. Atvars and M. Auzinsh, "A novel approach to quantitative spectroscopy of atoms in a magnetic field and applications based on an atomic vapor cell with $L=\lambda$ ", Appl. Phys. Lett. 93, 021119 (3p.) (2008). 